\title{
Modelagem da infiltração em solos com encrostamento superficial. Parte II: Condutividade hidráulica variando no tempo
}

\author{
João H. Zonta ${ }^{1}$, Mauro A. Martinez ${ }^{2}$, Demetrius D. da Silva ${ }^{2}$, \\ Fernando F. Pruski ${ }^{2} \&$ Marcelo R. dos Santos ${ }^{3}$
}

\begin{abstract}
RESU M O
Este trabalho objetivou avaliar o desempenho do modelo de Green-Ampt-M ein-Larson (GAML) na simulação do processo de infiltração em solos com encrostamento superficial. 0 s ensaios de infiltração foram realizados num Cambissolo com a utilização de simulador de chuvas, em solo nu. 0 valor da condutividade hidráulica na zona de transmissão (Kt) foi considerado igual a Kt* que, por sua vez, é igual à taxa de infiltração estável (Tie) multiplicada pelo fator $\mathrm{f}$, que representa o decaimento da Tie em função da energia cinética acumulada da chuva (EC), ou seja, o valor de Kt variando ao longo do tempo. 0 modelo GAML com o valor de Kt constante ao longo do tempo não obteve bom desempenho superestimando, na maioria dos casos, os valores da taxa de infiltração (Ti) enquanto com o uso de Kt* o modelo apresentou bom desempenho, em que os melhores resultados foram obtidos com a combinação de Kt* com o potencial matricial $\left(\varphi_{f}\right)$ calculado com uso da equação de Cecílio et al. (2007). O modelo de GAML com valor da condutividade hidráulica na zona de transmissão (Kt) variando no tempo apresentou bons resultados na simulação do processo de infiltração em solos sujeitos ao encrostamento superficial.
\end{abstract}

Palavras-chave: GAML,parâmetros, energia cinética da chuva

\section{Modelling of the soil water infiltration in crusting soil. Part II: Variable hydraulic conductivity over time}

\begin{abstract}
The soil crust affects significantly the soil water infiltration rate. Thus, the infiltration simulation models must somehow consider the effect of crust layer to obtain good results. The objective of this work was to evaluate the performance of the Green-Ampt-M ein-Larson (GAML) model to simulate the soil water infiltration for crusting soils. The trials were carried out in a Podzol Tb distrophic Haplic Cambisol, using a rain simulator, on a bare soil. The GAML model parameters were determined, being proposed for the hydraulic conductivity of transmission zone $(\mathrm{Kt})$ value the use of $\mathrm{Kt}^{*}$, which is equal to Tie $\mathrm{x} f$, where Tie is stable infiltration rate and $\mathrm{f}$ is a decrease factor of the Tie as a function of cumulative kinetic energy of rainfall (Ec), i.e., Kt value varying over time. The GAML model with constant Kt value over time did not provide good performance, overestimating the values of infiltration rate $(\mathrm{Ti})$ in most cases, where as the use of $\mathrm{Kt}^{*}$ the GAML model showed good performance, being the best results obtained by combination of Kt* with matric potential $\left(\varphi_{f}\right)$ values calculated by Cecílio et al. (2007) equation. The GAML model with the hydraulic conductivity in the transmission zone (Kt) value varying over time showed good results in simulation of the infiltration process in soils subjected to crusting.
\end{abstract}

Key words: GAML, parameters, kinetic energy of rainfall

\footnotetext{
${ }^{1}$ Embrapa Algodão, Rua 0 svaldo Cruz, 1143, Centenário, CEP 58428-095, Campina G rande, PB. Fone: (83) 3182-4355. E-mail: zonta@cnpa.embrapa.br 2 Departamento de Engenharia Agrícola/U FV, Campus U niversitário, s/n, Centro, CEP 36570-000, Viçosa, M G. Fone: (31) 3899-1910. E-mail: mmauro@ufv.br; david@ufv.br; ffpruski@ufv.br

3 Instituto Federal de Educação, Ciência e Tecnologia Baiano, IFBAIAN O, Distrito de Ceraíma, CEP 46430-000, Guanambi, BA. Fone: (77) $3493-2100$. E-mail: marrochas@yahoo.com.br
} 


\section{INTRODUÇÃO}

A infiltração de água no solo é um processo físico complexo, de difícil mensuração, sobremaneira nas condições normalmente encontradas de anisotropia e heterogeneidade dos solos, o qual ainda é influenciado pelas condições da superfície do solo, como a cobertura e o encrostamento superficial (Chowdary et al., 2006). A formação do encrostamento superficial é uma característica importante em solos de muitas regiões tropicais e subtropicais (Souza et al., 2007), de que vez que esta crosta pode apresentar espessura que varia de $0,1 \mathrm{~mm}$ até $50 \mathrm{~mm}$ (Brandão et al., 2006), com condutividade hidráulica do solo saturado menor que a da camada de solo subjacente, cuja redução pode ser superior a cem vezes o valor natural.

Desta forma, a simulação da infiltração de água no solo é um processo que exige diversas variáveis, muitas delas complexas de serem obtidas. Assim, o uso de um modelo mais simplificado matematicamente, que necessite de parâmetros de entrada facilmente determinados, torna-se interessante. Um modelo que vem sendo amplamente utilizado na estimativa da infiltração, incorporado a modelos matemáticos e computacionais baseados em processos, destinados à modelagem hidrológica e ao dimensionamento de sistemas hidroagrícolas, é o de Green \& Ampt modificado por Mein \& Larson, denominado modelo de Green-Ampt-Mein-Larson (GAML) (Liu et al., 2011).

Ressalta-se, porém, que a equação de GAML sem modificações nos seus parâmetros de entrada originais, não descreve satisfatoriamente o processo de infiltração de água no solo (Cecílio et al., 2003). Para a simulação em solos sem a presença de encrostamento superficial, diversos trabalhos já foram realizados visando definir metodologias de obtenção dos parâmetros de entrada do modelo, como os de Brakensiek \& Rawls (1983)e Cecílio et al. (2007). Sendo assim, este problema já dispõe de algumas soluções interessantes, as quais já apresentaram bons resultados em estudos anteriores (Mello, 2003; Cecílio et al., 2007; Zonta et al., 2010).

No entanto, quando se trabalha com solos sem cobertura em sua superfície e/ou sujeitos à formação de encrostamento superficial, mesmo com essas modificações em seus parâmetros de entrada, o modelo de GAML pode não simular bem o processo de infiltração tornando-se imprescindível, para melhorar seu desempenho, a inclusão desta camada de encrostamento (Zonta et al., 2010).

Alguns autores têm buscado realizar a análise do efeito do desenvolvimento do encrostamento superficial na infiltração e simular este processo (Moore, 1981; Brakensiek \& Rawls, 1983; Silva \& Kato, 1997; Souza et al., 2007); em muitos desses trabalhos foram encontrados bons resultados, com melhora significativa dos dados estimados. Moore (1981) desenvolveu, a partir do modelo de GAML, outro modelo capaz de simular o processo de infiltração em perfil de solo composto de duas camadas, permitindo não apenas a simulação em solos estratificados, mas também a inferência da camada de encrostamento superficial no modelo de GAML; entretanto, é grande a dificuldade na determinação das características hidráulicas da camada de encrostamento superficial.
Metodologias foram propostas para a determinação da condutividade hidráulica e espessura desta camada, porém muitas delas são de difícil aplicação no campo, visto a necessidade do uso de equipamentos eletrônicos e tecnologias de alto custo.

Mesmo com a consideração da camada de encrostamento superficial no modelo de GAML modificado por Moore (1981), os resultados obtidos na simulação podem não ser satisfatórios já que o modelo trabalha com parâmetros de entrada com valores constantes ao longo do tempo os quais, em muitos casos, deveriam ser considerados dinâmicos. Segundo Risse et al. (1995), a condutividade hidráulica do solo responde dinamicamente às mudanças no ambiente que circundam o solo, como mudanças na cobertura da superfície e efeitos da formação de encrostamento superficial devido à ação direta das gotas de chuva.

De acordo com Childs (1969), outro impasse no uso de modelos para solos estratificados surge quando a condutividade hidráulica saturada da camada superior é menor que a da camada inferior, como no caso em que ocorre encrostamento superficial, visto que a capacidade de infiltração é pouco afetada pelas características da camada inferior, sendo controlada pela camada superior; neste caso, é a camada de encrostamento que controla o processo de infiltração e o uso da média harmônica para o cálculo da condutividade hidráulica equivalente, como no modelo de GAML modificado por Moore (1981), não resultariam bons resultados.

Desta forma, uma alternativa seria trabalhar com o modelo de GAML para um único estrato de solo e utilizar, como parâmetro de entrada, o valor da condutividade hidráulica do solo saturado variando ao longo do tempo, relacionando esta variação a algum parâmetro que a influencie como, por exemplo, a energia cinética acumulada da chuva, conforme proposto por Silva \& Kato (1997) e Risse et al. (1995).

Outro fator a ser consideradoé a influência das precipitações antecedentes no processo de infiltração de água no solo já que, após um evento de precipitação, o solo contém elevado teor de água inicial e, em caso de solo descoberto pode apresentar, ainda, uma camada de encrostamento superficial resultante das ações das chuvas antecedentes, motivo por que a precipitação antecedente deve ser utilizada para descrever a diminuição da taxa de infiltração de água no solo, como discutido por Risse et al. (1995).

Devido aos fatos acima expostos, este trabalho teve como objetivo avaliar o desempenho do modelo de Green-AmptMein-Larson na simulação do processo de infiltração de água em solos com encrostamento superficial, considerando a condutividade hidráulica na zona de transmissão variável no tempo.

\section{MATERIAL E MÉTODOS}

Os ensaios de infiltração foram realizados na área experimental do Departamento de Engenharia Agrícola da UFV, em uma encosta próxima ao setor de Mecanização Agrícola. O solo da área foi classificado como Cambissolo Háplico Tb Distrófico, com declividade média de $8,0 \%$. Para a realização 
dos ensaios utilizou-se um simulador de chuvas do tipo pendular, sendo o mesmo automatizado permitindo, assim, a aplicação de precipitações com intensidade constante e variável no decorrer dos ensaios.

Os ensaios de infiltração consistiram na aplicação de chuva simulada seguindo-se quatro perfis de distribuição da intensidade de precipitação ao longo do tempo: constante, exponencial decrescente, duplo exponencial adiantado e atrasado (Figura 1), em solo sem cobertura.

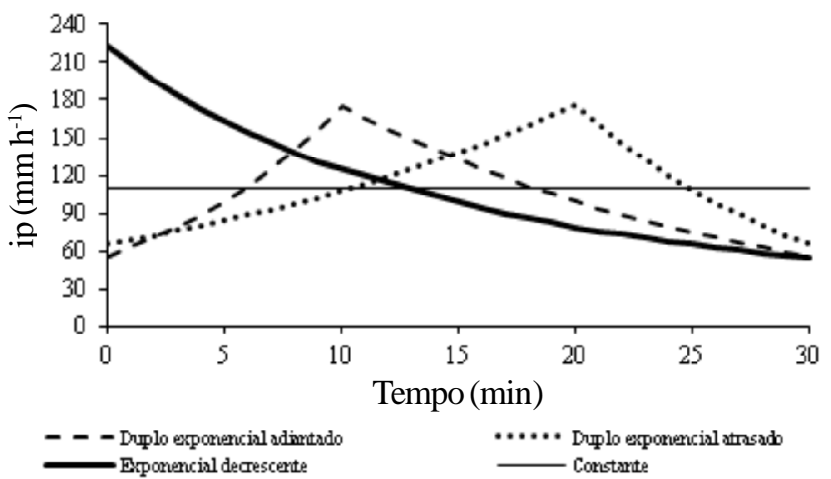

Figura 1. Perfis de precipitação utilizados no experimento

Os perfis de precipitação foram ajustados de modo que uma lâmina total de $55 \mathrm{~mm}$ fosse aplicada e a duração dos ensaios fosse de $30 \mathrm{~min}$, com intensidade máxima média de precipitação determinada o com uso da equação de intensidade-duração e frequência, para a região de Viçosa, MG, para um período de retorno de 15 anos, o atualmente mais indicado para dimensionamento de estruturas de conservação de solo e água, como terraceamento. Foram aplicadas, para cada perfil de precipitação, três chuvas sucessivas com intervalo de $24 \mathrm{~h}$, tempo em que o solo era mantido coberto, a fim de evitar a evaporação.

Foram feitas três repetições para cada perfil de precipitação, além de utilizado um valor médio das repetições na confecção das curvas da taxa de infiltração e infiltração acumulada experimentais, para cada perfil de precipitação, visando a uma comparação posterior aos dados simulados.

Foram coletados, para a aplicação do modelo de GAML na simulação do processo de infiltração de água no solo, os seguintes dados: teor de água no solo, com uso de sondas de TDR, no início, durante e no fim dos ensaios, nas profundidades de $10,20,30,40$ e $50 \mathrm{~cm}$; intensidade de precipitação (ip) e taxa de escoamento superficial e, por diferença, a taxa de infiltração (Ti) de água no solo e determinadas as características físicas do solo (Tabelas 1 e 2) usadas para determinação de alguns parâmetros de entrada do modelo.

O escoamento superficial produzido durante o ensaio foi conduzido para um recipiente de coleta, por meio de uma calha conectada a um tubo flexível de $50 \mathrm{~mm}$ de diâmetro. O recipiente de coleta era formado de reservatórios comunicantes construídos com dois tubos de PVC de $250 \mathrm{~mm}$ de diâmetro, localizados dentro de uma trincheira de 1,5 $\mathrm{m}$ de profundidade. Em um dos reservatórios foi instalado um linígrafo, denominado Thalimedes, que registrava a altura da lâmina de água ao longo do tempo, dentro do reservatório (Figura 2).
Tabela 1. M assa específica do solo ( $\rho)$, massa específica das partículas $\left(\rho_{\mathrm{r}}\right)$ porosidade total $(\varepsilon)$ e condutividade hidráulica do solo saturado $\left(\mathrm{K}_{0}\right)$ do perfil do solo e classe textural do solo em estudo

\begin{tabular}{|c|c|c|c|c|c|}
\hline \multirow{2}{*}{ Horizonte } & $\rho^{1}$ & $\rho_{r}^{2}$ & \multirow{2}{*}{$\underset{\mathrm{dm}^{3} \mathrm{dm}^{-3}}{\varepsilon}$} & \multirow{2}{*}{$\begin{array}{c}\mathrm{K}_{0}^{3} \\
\mathrm{~mm} \mathrm{~h}^{-1}\end{array}$} & \multirow{2}{*}{ Classe-Textura } \\
\hline & \multicolumn{2}{|c|}{$\mathrm{kg} \mathrm{m}^{-3}$} & & & \\
\hline A & 1,40 & 2,57 & 0,46 & 40,0 & Argilo-arenosa \\
\hline B & 1,37 & 2,58 & 0,47 & 20,0 & Argila \\
\hline
\end{tabular}

1 - Método do anel volumétrico; 2 - Método do balão volumétrico e 3 - Método do permeâmetro de carga constante

Tabela 2. Parâmetros do modelo de Brooks e Corey para os horizontes A e B do Cambissolo Háplico Tb Distrófico

\begin{tabular}{|c|c|c|c|}
\hline \multirow{2}{*}{ Horizonte } & \multicolumn{3}{|c|}{ Parâmetros } \\
\hline & $\lambda^{1}$ & $\theta_{\mathrm{r}}^{2}\left(\mathrm{~cm}^{3} \mathrm{~cm}^{-3}\right)$ & $\psi_{b}^{3}(\mathrm{~cm})$ \\
\hline$A$ & 0,1597 & 0,072 & 3,25 \\
\hline$B$ & 0,1358 & 0,076 & 6,38 \\
\hline
\end{tabular}

e 3 - Módulo do potencial matricial de entrada de ar

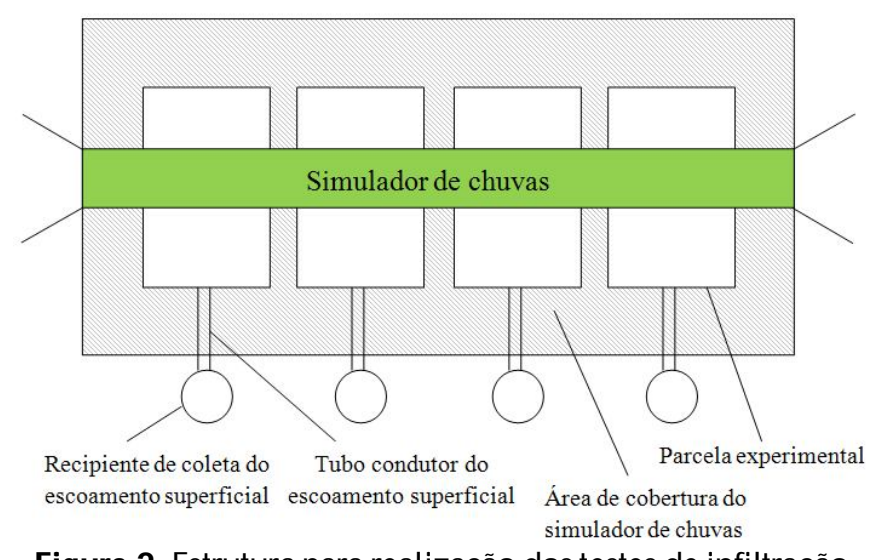

Figura 2. Estrutura para realização dos testes de infiltração

Amostras de solo deformadas e indeformadas foram coletadas nas camadas de 0-35 cm (Horizonte A) e $35-150 \mathrm{~cm}$ (Horizonte B) para determinação da classe textural e características físico-hídricas do solo, cujos resultados são apresentados nas Tabelas 1 e 2.

Aos dados experimentais da curva de retenção de água no solo foi ajustado o modelo de Brooks e Corey com uso do Software SWRC 2.0, cujos parâmetros são apresentados na Tabela 2.

O processo de infiltração de água no solo para as condições de solo sem cobertura foi simulado somente para as $2^{\mathrm{a}}$ e $3^{\mathrm{a}}$ aplicações da precipitação, visto que se considerou que o encrostamento superficial foi formado na $1^{\mathrm{a}}$ aplicação.

A capacidade de infiltração de água no solo (Ci) foi calculada com uso do modelo de GAML, Eq. 1:

$$
\mathrm{Ci}=\mathrm{K}_{\mathrm{t}}\left(1+\frac{\left|\varphi_{\mathrm{f}}\right|\left(\theta_{\mathrm{St}}-\theta_{\mathrm{i}}\right)}{\mathrm{I}(\mathrm{t})}\right)
$$

em que:

G - capacidade de infiltração $\mathrm{mm} \mathrm{h}^{-1}$

$\mathrm{Kt}$ - condutividade hidráulica do solo na zona de transmissão, $\mathrm{mm} \mathrm{h}^{-1}$ 
$\varphi_{\mathrm{f}}$ - potencial matricial do solo na frente de umedecimento, $\mathrm{mm}$

$\theta \mathrm{s}$ - teor de água do solo saturado, $\mathrm{m}^{3} \mathrm{~m}^{-3}$

$\theta \mathrm{i}$ - teor de água do solo no início do processo de infiltração, $\mathrm{m}^{3} \mathrm{~m}^{-3}$

$I(t)$ - infiltração acumulada no tempo, $\mathrm{mm}$

A capacidade de infiltração $(\mathrm{Ci})$ foi calculada a cada minuto e comparada ao valor da intensidade de precipitação (ip) enquanto no valor de $\mathrm{Ci}>$ ip, a taxa de infiltração (Ti) foi considerada igual à intensidade de precipitação (ip). Quando $\mathrm{Ci}<$ ip, a taxa de infiltração $(\mathrm{Ti})$ passa a ser considerada igual à capacidade de infiltração $(\mathrm{Ci})$, momento em que ocorre o empoçamento da superfície do solo. O valor da lâmina infiltrada em função do tempo, I(t), é calculada por meio da Eq. 2.

$$
\mathrm{I}(\mathrm{t})-\left(\Delta \theta_{1}\right) \varphi_{\mathrm{f} 1} \ln \left(1+\frac{\mathrm{I}(\mathrm{t})}{\left(\Delta \theta_{1}\right) \varphi_{\mathrm{f} 1}}\right)=\mathrm{Kt}_{1}\left(\mathrm{t}-\mathrm{tp}+\mathrm{tp}^{\prime}\right)
$$

em que:

$\mathrm{I}(\mathrm{t})$ - lâmina infiltrada no tempo t, em $\mathrm{mm}$

tp' - tempo requerido para infiltrar o volume equivalente à lâmina Ip em condições de empoçamento da superfície, em horas, obtido através da Eq. 2 substituindo-se $\mathrm{I}(\mathrm{t})$ por Ip e explicitando para tp', sendo Ip a lâmina infiltrada até o momento do empoçamento da superfície do solo, sendo igual ao valor de $\mathrm{I}$ quando $\mathrm{t}=\mathrm{tp}$.

Foram utilizadas adaptações para a determinação dos parâmetros de entrada originais do modelo de GAML. O valor de $\theta \mathrm{t}$ foi considerado como sendo igual ao teor de água da zona de transmissão $(\theta \mathrm{w})$, cujo valor médio foi de $0,418 \mathrm{~cm}^{3} \mathrm{~cm}^{-3}$, ou seja, $0,91 \theta$ s e para os valores de $\varphi_{\mathrm{f}}$ foram utilizadas duas propostas de adequação. Cecílio et al. (2007) propuseram a seguinte equação:

$$
\varphi_{\mathrm{f}}=\frac{\varphi_{\mathrm{b}}(2+3 \lambda)\left[\mathrm{K}_{\mathrm{r}}\left(\theta_{\mathrm{w}}\right)^{\frac{3 \lambda+1}{3 \lambda+2}}-\mathrm{K}_{\mathrm{r}}\left(\theta_{\mathrm{i}}\right)^{\left.\frac{3 \lambda+1}{3 \lambda+2}\right]}\right.}{\left[\mathrm{K}_{\mathrm{r}}\left(\theta_{\mathrm{w}}\right)-\mathrm{K}_{\mathrm{r}}\left(\theta_{\mathrm{i}}\right)\right][3 \lambda+1]}
$$

em que:

$\varphi_{\mathrm{b}}$ - potencial matricial de entrada de ar, $\mathrm{mm}$

$\varphi(q)$ - potencial matricial referente ao conteúdo de água $(\theta)$

Rawls \& Brakensiek (1983) propuseram uma metodologia na qual $\varphi_{\mathrm{f}}$ é obtido através das características físicas do solo, conforme a Eq. 4.

$$
\begin{gathered}
\varphi_{\mathrm{f}}=0,01 \mathrm{e}^{\alpha} \\
\alpha=6,531-7,326 \phi+15,8 \mathrm{C}^{2}+3,809 \phi^{2}+3,44 \mathrm{SC} \\
-4,989 \mathrm{~S} \phi+16,1 \mathrm{~S}^{2} \phi^{2}+16 \mathrm{C}^{2} \phi^{2}-13,6 \mathrm{~S}^{2} \mathrm{C} \\
-34,8 \mathrm{C}^{2} \phi-7,99 \mathrm{~S}^{2} \phi
\end{gathered}
$$

em que:

$\varphi_{\mathrm{f}} \quad$ - potencial matricial na frente de umedecimento, $\mathrm{mm}$

$\phi \quad$ - porosidade total do solo, $\mathrm{m}^{3} \mathrm{~m}^{-3}$

$\mathrm{S}$ - teor de areia (decimal) e C o teor de argila (decimal)

Para adequação do valor da condutividade hidráulica na zona de transmissão $(\mathrm{Kt})$ para solos com encrostamento, foi proposta a seguinte equação:

$$
\mathrm{Kt}^{*}=\mathrm{Tie} \times \mathrm{f}
$$

em que:

Tie - taxa de infiltração estável, $\mathrm{mm} \mathrm{h}^{-1}$

f - fator de decaimento da taxa de infiltração estável em função da energia cinética acumulada da chuva, adimensional

Para obtenção do fator $\mathrm{f}$ foi utilizado o valor da Tie obtida em solo com cobertura $\left(60 \mathrm{~mm} \mathrm{~h}^{-1}\right)$, ou seja, sem impacto das gotas de chuva e os valores da Tie obtidos nas $2^{\mathrm{a}}$ e $3^{\mathrm{a}}$ aplicações de precipitação, ou seja, para vários valores de energia cinética acumulada da chuva (Ec) aplicada na superfície do solo sem cobertura, isto é, com impacto direto das gotas de chuva. $\mathrm{O}$ índice $\mathrm{f}$ para cada valor de energia cinética acumulada da chuva (Ec) foi calculado, então, pela Eq. 6:

$$
\mathrm{f}=\frac{\mathrm{Tie}(\mathrm{Ec})}{\text { Tie }_{\text {cobertura }}}
$$

em que:

Tie (Ec) - taxa de infiltração estável obtida para um valor de energia cinética acumulada da chuva, em solo sem cobertura, $\mathrm{mm} \mathrm{h}^{-1}$

Tie $_{\text {cobertura }}$ - taxa de infiltração estável obtida em solo com cobertura, $\mathrm{mm} \mathrm{h}^{-1}$

A energia cinética acumulada da chuva (Ec) para cada um dos perfis de precipitação aplicados, foi calculada conforme metodologia proposta por Wischmeier \& Smith:

$$
\mathrm{Ec}=0,119+0,0873 \log (\mathrm{ip})
$$

em que:

Ec - energia cinética das gotas de chuvas, $\mathrm{MJ} \mathrm{ha}^{-1} \mathrm{~mm}^{-1}$

ip - intensidade de precipitação, $\mathrm{mm} \mathrm{h}^{-1}$

A Eq. 8 foi aplicada a cada intervalo de $1 \mathrm{~min}$, no qual a intensidade de precipitação era constante e o resultado obtido multiplicado pela lâmina precipitada em cada intervalo e, em seguida, realizada a soma de todos os valores para cada perfil de precipitação obtendo-se, com isto, a energia cinética acumulada para cada perfil. Nas aplicações sucessivas foi considerado que, inicialment, já havia sido aplicada a energia cinética acumulada da aplicação anterior; assim, ao final da terceira aplicação tem-se como energia cinética acumulada o total das três aplicações. 
Com os valores de f determinados para cada valor de energia cinética acumulada da chuva (Ec) foi ajustada uma equação do tipo exponencial (Eq. 8) que descreve o decaimento na taxa de infiltração estável (Tie) em função da energia cinética acumulada da chuva (Ec).

$$
\mathrm{f}=\alpha \cdot \mathrm{e}^{-\beta \cdot \mathrm{Ec}}+\mathrm{C}
$$

em que:

$\alpha, \beta$ e C - parâmetros das equações

Na Tabela 3 são apresentados os valores dos parâmetros da equação ajustada e o valore de $\mathrm{R}^{2}$.

Tabela 3. Parâmetros da equação ajustada e coeficiente de determinação $\left(R^{2}\right)$

\begin{tabular}{ccccc}
\hline \multirow{2}{*}{ Equação } & \multicolumn{3}{c}{ Parâmetros } & \multirow{2}{*}{$\mathbf{R}^{2}$} \\
\cline { 2 - 4 } & $\boldsymbol{\alpha}$ & $\boldsymbol{\beta}$ & $\mathbf{C}$ & \\
Exponencial & $0,842^{*}$ & $0,0061^{*}$ & $0,157^{*}$ & 0,989 \\
\hline Significativo a $5 \%$ de probabilidade & & &
\end{tabular}

Na Figura 3 se apresenta o gráfico, relacionando-se f em função de Ec com a curva do tipo exponencial ajustada.

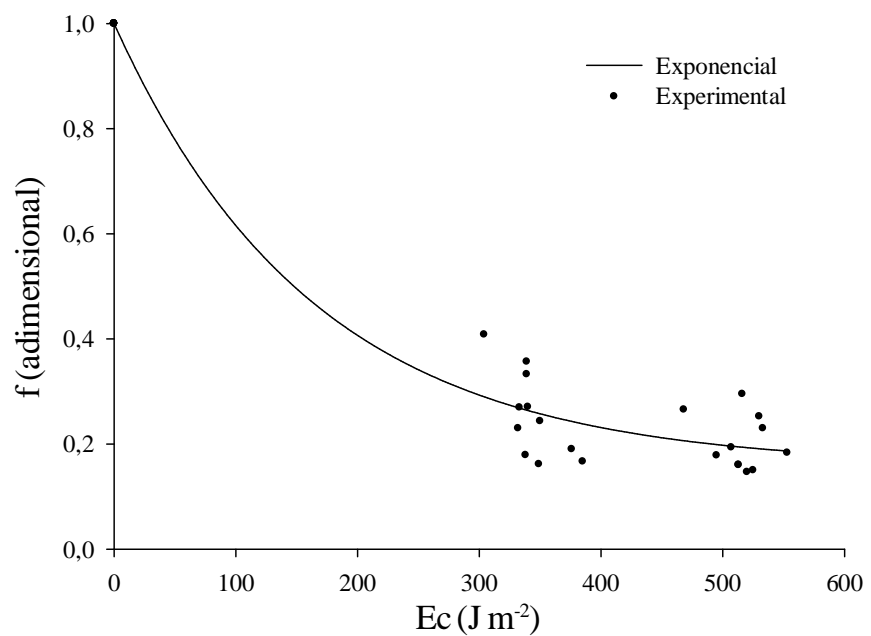

Figura 3. Valores do decaimento da Tie (f) em função da energia cinética acumulada da chuva

Na Tabela 4 são apresentadas as combinações testadas para os parâmetros de entrada do modelo de GAML.

Fez-se a avaliação do desempenho do modelo de GAML comparando-se as curvas experimentais com as simuladas da taxa de infiltração e lâmina infiltrada; para isto, utilizou-se o índice de confiança (c) obtido pelo produto entre o coeficiente de correlação de Pearson (r) e o índice de concordância (d), obtido pela Eq. 9 .

$$
\mathrm{d}=1-\frac{\sum_{\mathrm{i}=1}^{\mathrm{J}}\left(\left|\mathrm{O}_{\mathrm{i}}-\mathrm{E}_{\mathrm{i}}\right|\right)^{2}}{\sum_{\mathrm{i}=1}^{\mathrm{J}}\left(\left|\mathrm{E}_{\mathrm{i}}=\overline{\mathrm{O}}\right|+\left|\mathrm{O}_{\mathrm{i}}-\overline{\mathrm{O}}\right|\right)^{2}}
$$

em que:
J - número de observações
Oi - valor observado experimentalmente
Ei - valor estimado pelo modelo
$\overline{\mathrm{O}}$ - média dos valores observados experimentalmente

O desempenho do modelo avaliado pelo uso do índice de confiança (c), foi classificado conforme apresentado na Tabela 5.

Tabela 5. Análise do desempenho do modelo com base no índice de confiança

\begin{tabular}{cl}
\hline Valor de c & Desempenho \\
$>0,85$ & Ótimo \\
0,76 a 0,85 & Muito bom \\
0,66 a 0,75 & Bom \\
0,61 a 0,65 & Mediano \\
0,51 a 0,60 & Sofrível \\
0,41 a 0,50 & Mau \\
$\leq 0,40$ & Péssimo \\
\hline
\end{tabular}

\section{RESULTADOS E DISCUSSÃO}

O desempenho do modelo de GAML na simulação da taxa de infiltração (Ti) e infiltração acumulada (I) consideradas as diferentes combinações de seus parâmetros de entrada, para as $2^{\mathrm{a}}$ e $3^{\mathrm{a}}$ aplicações da precipitação em intervalos de $24 \mathrm{~h}$, com os quatro perfis de precipitação aplicados, é apresentado nas Tabelas 6 e 7. Nas Figuras 4 a 7 se encontram os gráficos das curvas da taxa de infiltração (Ti) e lâmina infiltrada (I) ao longo do tempo, medidas e simuladas com as diferentes combinações de parâmetros de entrada do modelo de GAML, para as $2^{\mathrm{a}}$ e $3^{\mathrm{a}}$ aplicações da precipitação, para os perfis de precipitação constante e duplo exponencial adiantado.

De acordo com os resultados apresentados nos Quadros 7 e 8 nota-se, para todos os perfis de precipitação aplicados, que a combinação $a$ apresentou os melhores resultados, com desempenho pelo índice de confiança (c) superior a bom para Ti e I, em todos os casos; para esta combinação são utilizados os valores de $\mathrm{Kt}$ variando ao longo do tempo ( $\left.\mathrm{Kt}^{*}\right)$. O valor de $\varphi_{\mathrm{f}}$ é calculado com uso da equação proposta por Cecílio et al. (2007) e os valores de $\theta$ t são iguais a $\theta \mathrm{w}$, resultados que demonstram que o uso do valor de Kt variando ao longo do tempo em conjunto com o valor de $\varphi_{\mathrm{f}}$ calculado com uso da equação proposta por Cecílio et al. (2007) acarreta bom desempenho na simulação do modelo de GAML para solos com encrostamento superficial.

Tabela 4. Combinações de proposições utilizadas na análise dos dados

\begin{tabular}{cclc}
\hline Combinação & \multicolumn{1}{c}{ Kt } & \multicolumn{1}{c}{$\boldsymbol{\varphi}_{\mathbf{f}}$} & \multicolumn{1}{c}{$\boldsymbol{\theta}_{\text {st }}$} \\
a & Kt* & Cecílio et al. (2007) & $\theta$ w - Slack (1980) \\
b & Kt* & Rawls \& Brakensiek (1983) & $\theta \mathrm{w}-$ Slack (1980) \\
c & Tie $\left(60 \mathrm{~mm} \mathrm{~h}^{-1}\right)$ & Cecílio et al. (2007) & $\theta \mathrm{w}-$ Slack (1980) \\
d & Tie $\left(60 \mathrm{~mm} \mathrm{~h}^{-1}\right)$ & Rawls \& Brakensiek (1983) & $\theta \mathrm{w}-$ Slack (1980) \\
\hline
\end{tabular}


Tabela 6. Desempenho do modelo de GAM L na simulação da taxa de infiltração (Ti) e lâmina infiltrada (I) com base no índice c para a 2 a aplicação da precipitação

\begin{tabular}{ccllll}
\hline \multirow{2}{*}{ Combinação } & Parâmetro & \multicolumn{3}{c}{ Perfil de precipitação } \\
\cline { 3 - 6 } & & Constante & Exponencial & Adiantado & Atrasado \\
a & $\mathrm{Ti}$ & Ótimo & Muito Bom & Muito Bom & Bom \\
& $\mathrm{I}$ & Ótimo & Ótimo & Ótimo & Ótimo \\
$\mathrm{b}$ & $\mathrm{Ti}$ & Mau & Sofrível & Mau & Mediano \\
& $\mathrm{I}$ & Sofrível & Muito Bom & Mediano & Muito Bom \\
$\mathrm{C}$ & $\mathrm{Ti}$ & Mediano & Bom & Péssimo & Péssimo \\
& $\mathrm{I}$ & Muito Bom & Péssimo & Bom & Mau \\
$\mathrm{d}$ & $\mathrm{Ti}$ & Péssimo & Mau & Péssimo & Péssimo \\
& $\mathrm{I}$ & Ótimo & Sofrível & Ótimo & Bom \\
\hline
\end{tabular}

Tabela 7. Desempenho do modelo de GAM L na simulação da taxa de infiltração (Ti) e lâmina infiltrada (I) com base no índice c para 3a aplicação da precipitação

\begin{tabular}{ccllll}
\hline \multirow{2}{*}{ Combinação } & \multirow{2}{*}{ Parâmetro } & \multicolumn{4}{c}{ Perfil de precipitação } \\
\cline { 3 - 6 } & & Constante & Exponencial & Adiantado & Atrasado \\
a & $\mathrm{Ti}$ & Muito Bom & Bom & Ótimo & Bom \\
& $\mathrm{I}$ & Ótimo & Ótimo & Ótimo & Ótimo \\
b & $\mathrm{Ti}$ & Péssimo & Sofrível & Péssimo & Péssimo \\
& $\mathrm{I}$ & Sofrível & Bom & Mediano & Mediano \\
& $\mathrm{Ti}$ & Mau & Bom & Péssimo & Péssimo \\
& $\mathrm{I}$ & Mediano & Péssimo & Sofrível & Sofrível \\
& $\mathrm{Ti}$ & Péssimo & Mau & Péssimo & Péssimo \\
& $\mathrm{T}$ & Ótimo & Sofrível & Bom & Bom \\
\hline
\end{tabular}

A.

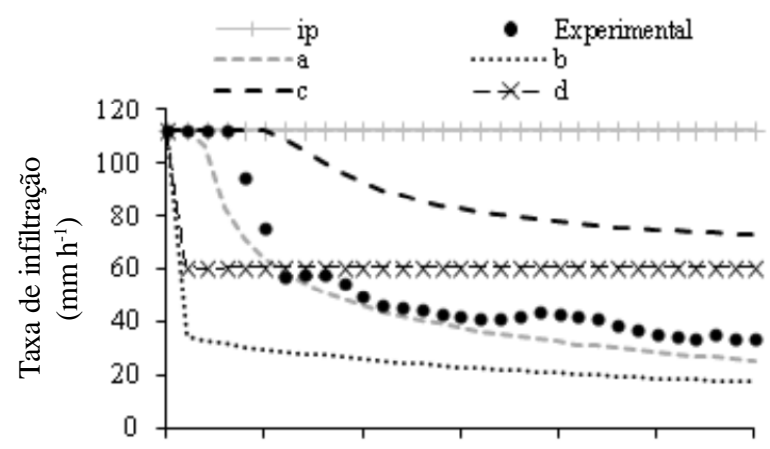

B.

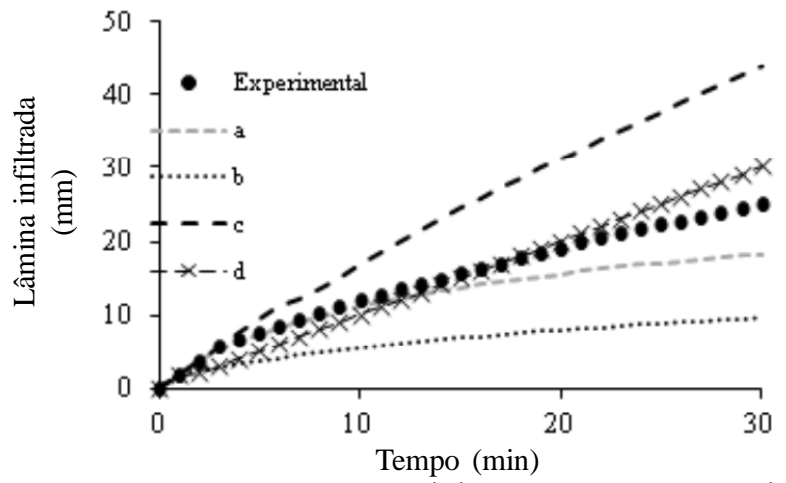

Figura 4. Taxa de infiltração (A) e lâmina infiltrada (B) medidas e estimadas pelo modelo de G AM L usando-se diferentes combinações de seus parâmetros de entrada, 2a aplicação e perfil de precipitação constante

Observa-se, nas Figuras 4 a 7, que combinação a não apresentou comportamento comum de subestimar ou superestimar os valores de Ti e I ao longo do tempo mas sempre apresentou pequenos desvios em relação aos valores
A.

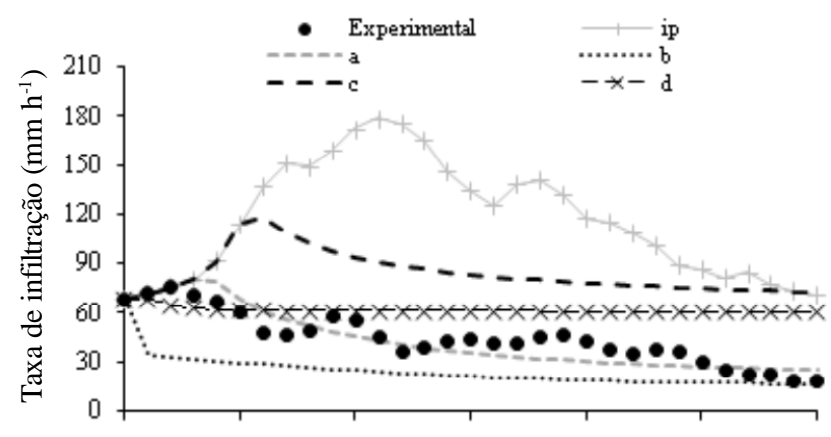

B.

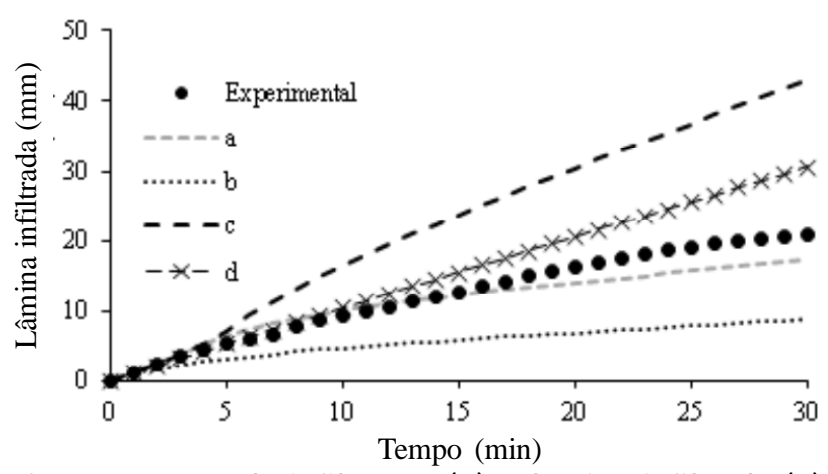

Figura 5. Taxa de infiltração (A) e lâmina infiltrada (B) medidas e estimadas pelo modelo de GAML usando-se diferentes combinações de seus parâmetros de entrada, $2^{a}$ aplicação, perfil de precipitação duplo exponencial adiantado

observados sendo que, de forma geral, referidas curvas foram as que indicaram comportamento mais próximo dos observados experimentalmente, para os quatro perfis de precipitação aplicados. 
A.

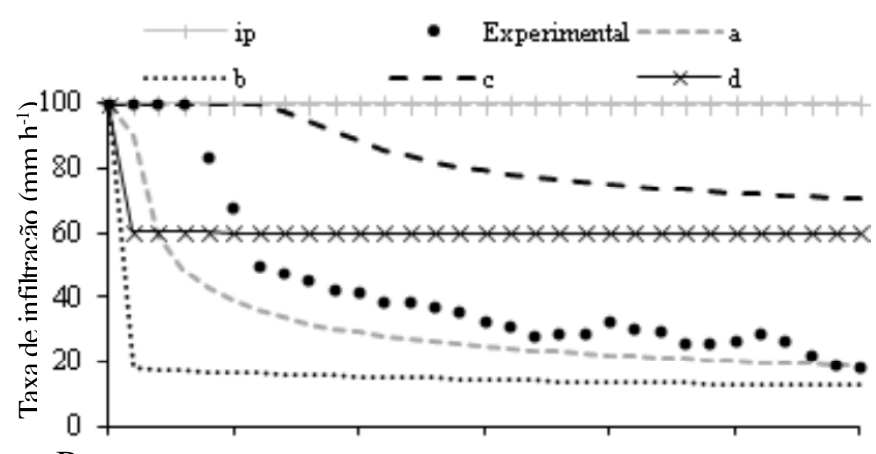

B.

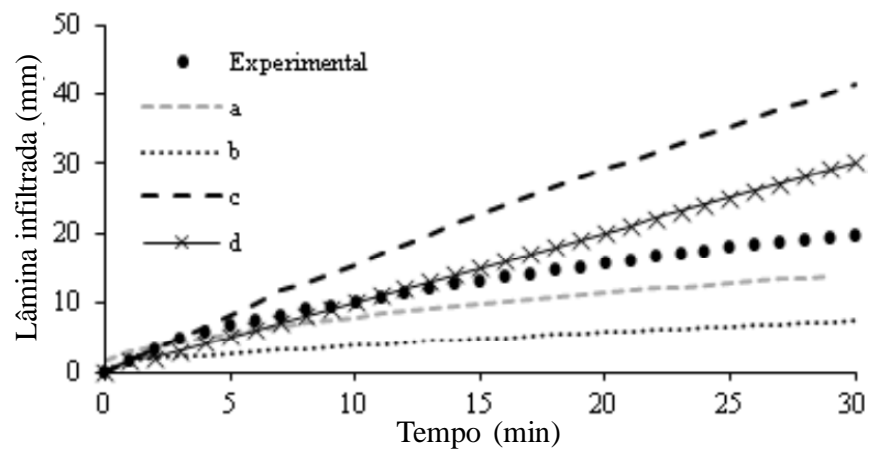

Figura 6. Taxa de infiltração $(A)$ e lâmina infiltrada $(B)$ medidas e estimadas pelo modelo de GAM L usando-se diferentes combinações de seus parâmetros de entrada, 3a aplicação, perfil de precipitação constante

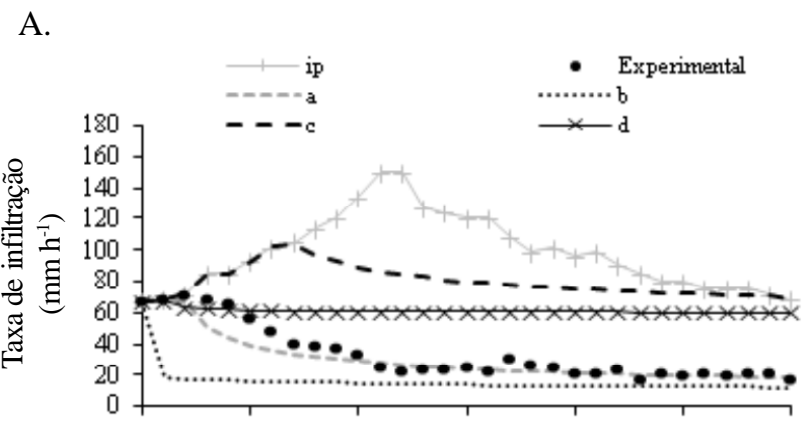

B.

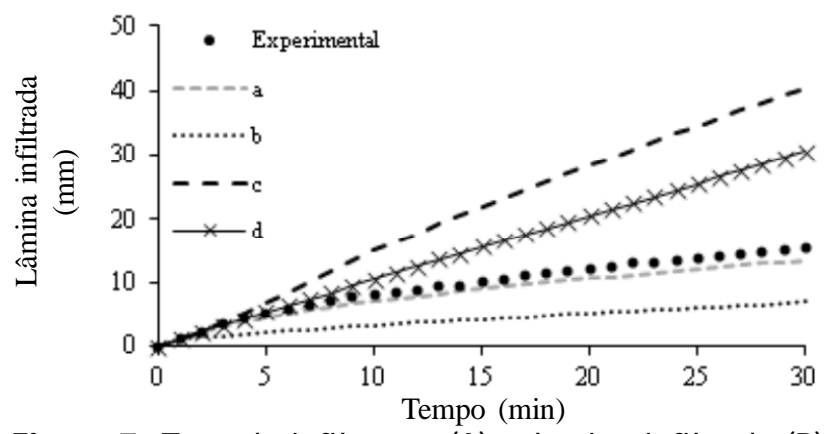

Figura 7. Taxa de infiltração (A) e lâmina infiltrada (B) medidas e estimadas pelo modelo de GAML usando-se diferentes combinações de seus parâmetros de entrada, 3a aplicação, perfil de precipitação duplo exponencial adiantado

Além disso, esta combinação foi a que melhor estimou o início do processo de infiltração, simulando bem o momento do início do escoamento superficial (tp) tal como também o valor final da Ti (Figuras 4 a 7). Segundo Risse et al. (1995), a energia cinética da chuva $(\mathrm{Ec})$ apresenta alta correlação com a infiltração de água no solo, visto que influencia altamente na formação e espessura da camada de encrostamento formada e ainda apresenta alta correlação com outros parâmetros, como o tempo de empoçamento da superfície (tp).

A explicação dos bons resultados obtidos com a combinação a é o uso do valor de $\mathrm{Kt}^{*}$ como parâmetro de entrada visto que, nele, a variação da condutividade hidráulica do solo saturado é função da energia cinética acumulada da chuva. Diversos autores, como Kim \& Chung (1994) afirmam que a variação temporal nos valores da condutividade hidráulica do solo pode ser expressa como função da energia cinética acumulada da chuva.

No presente trabalho a correção no valor de Kt foi realizada através do ajuste exponencial do decaimento da Tie, em função da energia cinética acumulada da chuva, apresentando bons resultados de acordo, portanto, com os trabalhos de Chu et al. (1986) e Risse et al. (1995).

As combinações c e d, que utilizam o valor da Tie constante ao longo do tempo como valor de Kt não apresentarm, de forma geral, bons resultados, independente do método utilizado para determinação do parâmetro $\varphi_{\mathrm{f}}$ e do perfil de precipitação, com índice de confiança c apresentando desempenho acima de bom, mas somente em 12,5 e $50 \%$ dos casos para Ti e I, respectivamente, sendo os valores de Ti e I superestimados, com a combinação d subestimando os valores de Ti e I somente nos momentos iniciais, devido à queda brusca nos valores de Ti decorrente do baixo valor de $\varphi_{f}$ (Figuras 4 a 7).

Savabi et al. (1995) encontraram superestimativa da taxa de infiltração simulada com o modelo de GAML, sendo este resultado atribuído a erros na determinação do valor de $\mathrm{Kt}$ para as condições de encrostamento superficial, apesar de os valores terem sido determinados em função do tipo de cobertura do solo; apesar disto, os autores trabalharam com valores constantes de Kt ao longo do tempo podendo, esta cobertura, ser sua fonte de erro, comprovando a importância em se trabalhar com os valores de Kt variando ao longo do tempo quando se tem solos sujeitos ao encrostamento superficial, como foi o caso deste experimento. Ainda segundo Wilson \& Oduro (2004), a condutividade hidráulica é o parâmetro ao qual o modelo de GAML apresenta maior sensibilidade.

Se comparados os resultados das combinações a e c, cuja diferença está no parâmetro Kt, nota-se que a combinação c, na qual o valor de Kt é constante ao longo do tempo, apresentou desempenho acima de bom mas somente em $25 \%$ dos casos para Ti e I; esses resultados demonstram, para o caso de solos expostos ao encrostamento superficial, que o valor de Kt deve ser corrigido a fim de se incluir seu efeito no modelo.

Segundo Risse et al. (1995), dinamicamente a condutividade hidráulica do solo responde às mudanças no ambiente que circundam o solo, como mudanças na cobertura da superfície e efeitos da formação de encrostamento superficial em virtude da ação direta das gotas de chuva; desta forma, os parâmetros do modelo de GAML deveriam mudar, por exemplo, para cada evento de precipitação contínuo, explicando os bons 
resultados obtidos por combinações nas quais o valor de $\mathrm{Kt}$ varia em função da energia cinética da chuva aplicada.

A combinação b não apresentou bons resultados, com desempenho abaixo de mediano em 100 e 62,5\% dos casos para Ti e I, respectivamente; esses resultados podem ser atribuídos ao uso da equação proposta por Rawls \& Brakensiek (1983) para o cálculo de $\psi_{\mathrm{f}}$, que é calculado em função da textura do solo obtido com uso de uma equação empírica, passível de não ser aplicável para as condições deste experimento, fato que se torna claro, visto que os demais parâmetros de entrada do modelo são os mesmos da combinação a.

A combinação b subestimou. durante todo o intervalo de tempo, os valores de Ti e I, subestimativa que foi mais acentuada no início do processo de infiltração (Figuras 4 a 7); este evento devido ao baixo valor de $\psi_{\mathrm{f}}$ calculado com uso da equação proposta por Rawls \& Brakensiek (1983), na faixa de $2 \mathrm{~mm}$; com isto, nota-se que nas curvas simuladas com a combinação b a capacidade de infiltração teve queda muito acentuada logo no início do evento de precipitação e, em sendo assim, ocorreu o escoamento superficial desde o começo do teste, com valor de tp sendo subestimado.

Se comparadas as duas equações para o cálculo do potencial matricial da água no solo ( $\ddot{\mathrm{o}}_{\mathrm{f}}$ ) para a equação proposta por Rawls \& Brakensiek (1983) constata-se que o valor se mantém na faixa de $2 \mathrm{~mm}$ enquanto para a equação proposta por Cecílio et al. (2007) o valor foi, em média, $110 \mathrm{~mm}$.

Diversos autores, como Chu et al. (1986), citam que os valores de $\varphi_{\mathrm{f}}$ para obtenção de bons resultados na simulação do processo de infiltração com uso do modelo de GAML variam de 70 a $130 \mathrm{~mm}$. Kunze \& Shayya (1993) encontraram valores de $\varphi_{\mathrm{f}}$ na faixa de 70 a $640 \mathrm{~mm}$ com uso de dados simulados ajustados aos dados experimentais, enquanto Wells et al. (1986) encontraram valores de $\varphi_{\mathrm{f}}$ na faixa de $200 \mathrm{~mm}$.

Como o valor da Ti tendeu a tornar-se estável logo no início do evento de precipitação, em virtude do encrostamento superficial, a combinação b simula bem os valores de $\mathrm{Ti}$, principalmente no terço final do ensaio de infiltração, resultados que podem ser atribuídos ao fato de o valor de $\mathrm{Kt}^{*}$ ser igual a Tie $\mathrm{x}$ f e; como o valor $\varphi_{\mathrm{f}}$ é muito pequeno, o que torna o gradiente hidráulico praticamente unitário, o valor de $\mathrm{Ti}$ simulado tende para $\mathrm{Kt}^{*}$ que, na verdade, é o valor de Tie ajustada em função da energia cinética acumulada da chuva.

Yu (1999) discorda do uso de equações empíricas para o cálculo de parâmetros físicos do solo. Um dos problemas em se trabalhar com a equação proposta por Rawls \& Brakensiek (1983) para o cálculo de $\ddot{o}_{f}$ é que esta resulta em valores muito pequenos que, no caso do solo em estudo, foi na faixa de $2 \mathrm{~mm}$, ou seja, desde o início do processo de infiltração o solo já apresenta gradiente unitário do potencial total de água no solo acarretando, em muitos casos, na subestimativa dos valores $\mathrm{Ti}$ e I ao longo do tempo, conforme relatado por Zonta et al. (2010).

Para todas as combinações os valores de I foram, no geral, melhor simulados que os valores de $\mathrm{Ti}$, corroborando com os resultados obtidos por Cecílio et al. (2003) e Zonta et al. (2010). $\mathrm{Yu}$ (1999) afirma que o modelo de GAML apresenta boa eficiência na simulação do total infiltrado e subestima a taxa de infiltração, sobretudo em eventos com alta intensidade de precipitação, superestimando os valores da taxa de escoamento superficial, concordando com os resultados acima descritos, principalmente pelo fato do autor ter trabalhado com equações baseadas na textura do solo para obtenção dos parâmetros de entrada do modelo.

Assim, pode-se observar que quando a variável de interesse a ser determinada é o total infiltrado, o modelo de GAML pode ser usado com maior confiabilidade, como o caso de dimensionamento de estruturas conservacionistas, como terraços construídos em nível; quando, no entanto, o objetivo é a determinação da taxa de infiltração, o modelo deve ser usado com algumas restrições sendo que o cálculo do potencial matricial na frente de umedecimento a partir da textura do solo e a condutividade hidráulica na zona de transmissão com valor constante ao longo do tempo, não são recomendados.

Outro fato importante é que, para um evento de precipitação isolado, um único valor para o parâmetro Kt do modelo de GAML pode ser determinado e acarretar bons resultados simulados, conforme apresentado por diversos autores, como Mello (2003), Cecílio et al. (2003) eZonta et al. (2010). Entretanto, para eventos contínuos de precipitação o valor de Kt precisa ser determinado com precisão tanto no início do processo de infiltração como na sua variação ao longo do tempo. Risse et al. (1995) afirmaram, trabalhando com o modelo WEPP, que a condutividade hidráulica do solo saturado deve ser variável ao longo do tempo sendo, neste caso, em função das variações na superfície do solo como, por exemplo, o encrostamento superficial, vindo de encontro aos resultados obtidos neste experimento.

\section{Conclusões}

1. O modelo de GAML com valor da condutividade hidráulica na zona de transmissão (Kt) variando no tempo, apresentou bons resultados na simulação do processo de infiltração em solos sujeitos ao encrostamento superficial.

2. O uso do potencial matricial na frente de umedecimento $\left(\varphi_{\mathrm{f}}\right)$ calculado a partir da textura do solo leva a subestimativa dos valores de Ti e I ao longo do tempo se combinado com a condutividade hidráulica na zona de transmissão variável no tempo.

\section{Agradecimentos}

À Fundação de Amparo à Pesquisa do Estado de Minas Gerais (FAPEMIG) pelo apoio e financiamento do projeto de pesquisa.

\section{LITERATURA CITADA}

Brakensiek, D. L.; Rawls, W. J. Agricultural management effects on soil water processes Part II: Green and Ampt parameters for crusting soils. Transactions of the ASAE, v.26, p.17531757, 1983. 
Brandão, V. S.; Silva, D. D.; Ruiz, H. A.; Pruski, F. F.; Schaefer, C. E. G. R.; Martinez, M. A.; Menezes, S. J. M. C. Resistência hidráulica da crosta formada em solos submetidos a chuvas simuladas. Revista Brasileira de Ciência do Solo, v.30, p.1321, 2006.

Cecílio, R. A.; Martinez, M. A.; Pruski, F. F.; Silva, D. D.; Ataíde, W .F. Substituição dos parâmetros do modelo de GreenAmpt-Mein-Larson para estimativa da infiltração em alguns solos do Brasil. Revista Brasileira de Ciência do Solo, v.31, p.1141-1151, 2007.

Cecílio, R. A.; Silva, D. D.; Pruski, F. F.; Martinez, M. A. Modelagem da infiltração de água no solo sob condições de estratificação utilizando-se a equação de Green-Ampt. Revista Brasileira de Engenharia Agrícola e Ambiental, v.7, p.415-422, 2003.

Childs, E. C. An introduction to the physical basis of soil water phenomena. London/New York: John Wiley e Sons, 1969. 281p.

Chowdary, V. M.; Rao, M. D.; Jaiswal, C. S. Study of infiltration process under different experimental conditions. Agricultural Water Management, v.83, p.69-78, 2006.

Chu, S. T.; Onstad, C. A.; Rawls, W. J. Field evaluation of layered Green-Ampt model for transient crust conditions. Transactions of the ASAE, v.29, p1268-1272, 1986.

Kim, S. J.; Chung, H. W. Field evaluation of layered GreenAmpt infiltration model considering temporal variation of physical properties. Transacions of the ASAE, v.37, p.1845$1852,1994$.

Kunze, R. J.; Shayya, W. H. Assessing the constancy of the potential term in the Green and Ampt infiltration equation. Transacions of the ASAE, v.36, p.1093-1098, 1993.

Liu, G.; Craig, J. R.; Soulis, E. D. Applicability of the GreenAmpt infiltration model with shallow boundary conditions. Journal of Hydrologic Engineering, v.16, p.266-273, 2011.

Mello, L. T. A. Avaliação de metodologias para obtenção dos parâmetros do modelo de Green-Ampt modificado por Mein e Larsson. Viçosa: UFV, 2003. 77p. Dissertação Mestrado
Moore, I. D. Infiltration equations modified for surface effects. Journal of the Irrigation and Drainage Division, v.107, p.71$86,1981$.

Rawls, W. J.; Brakensiek, D. L. A procedure to predict Green and Ampt infiltration parameters. In: ASAE Conference on Advances in Infiltration, 1983, Chicago. Proceedings.... Chicago: ASAE, 1983. p.102-112.

Risse, L. M.; Nearing, M. A.; Zhang, X. C. Variability in GreenAmpt effective hydraulic conductivity under fallow conditions. Journal of Hydrology, v. 169, p.1-24, 1995.

Savabi, M. R.; Rawls, W. J.; Knight, R. W. Water erosion prediction project (WEPP) rangeland hydrology component evaluation on a Texas range site. Journal of Range Management, v.48, p.535-541, 1995.

Silva, C. L.; Kato, E. Efeito do selamento superficial na condutividade hidráulica saturada da superfície de um solo sob cerrado. Pesquisa Agropecuária Brasileira, v.32, p.213220, 1997.

Slack, D. C. Modeling infiltration under moving sprinkler irrigation systems. Transactions of the ASAE, v.23, p.596-600, 1980.

Souza, E. S.; Antonino, A. C. D.; Lima, J. R. S.; Gouveia Neto, G. C.; Silva, J. M.; Silva, I. F. Efeito do encrostamento superficial nas propriedades hidráulicas de um solo cultivado. Revista Brasileira de Ciências Agrárias, v.2, p.69-74, 2007.

Wells, L. G; Ward, A. D.; Moore, I. D.; Phillips, R. E. Comparison of four models in characterizing through surface mine profiles. Transactions of the ASAE, v.29, p.785-793, 1986.

Wilson, B. N.; Oduro, P. Analytical sensitivity coefficients for the GAML infiltration model. Transactions of the ASAE, v.47, p.479-484, 2004.

$\mathrm{Yu}, \mathrm{B}$. A comparison of the Green-Ampt and a spatially variable infiltration model for natural storm events. Transactions of the ASAE, v.42, p.88-97, 1999.

Zonta, J. H.; Martinez, M. A.; Pruski, F. F.; Silva, D. D.; Montebeller, C. A. Adequação dos parâmetros do modelo de Green-AmptMein-Larson em condições de campo. Revista Brasileira de Engenharia Agrícola eAmbiental, v.14, p.1021-1029, 2010. 